\title{
ESTUDO DA CINÉTICA DE HIDRATAÇÃO E PROPRIEDADES DO GRÃO DE ARROZ DURANTE O PROCESSO DE PARBOILIZAÇÃO
}

\author{
T. C. VOLPE ${ }^{1}$; A. M. R. SANTOS ${ }^{2}$; L. M. M. JORGE 3 ; R. M. M. JORGE ${ }^{4}$ \\ ${ }^{1,4}$ Programa de Pós-Graduação em Engenharia de Alimentos. Departamento de Engenharia \\ Química - Universidade Federal do Paraná (UFPR), Av. Cel. Francisco H dos Santos, s/n, Jardim \\ das Américas, CEP: 81530-900 - Curitiba - PR - Brasil \\ ${ }^{2}$ Departamento de Engenharia Química, Universidade Federal do Paraná (UFPR) \\ ${ }^{3}$ Departamento de Engenharia Química, Universidade Estadual de Maringá (UEM), CEP 87020- \\ 900 - Maringá - PR - Brasil \\ *E-mail para contato: thaisavolpe@hotmail.com, rjorge@ufpr.br
}

\begin{abstract}
RESUMO - O objetivo deste trabalho foi estudar o fenômeno da hidratação de grãos de arroz da variedade IRGA-417 para diferentes temperaturas no processo de parboilização tendo por finalidade, compreender o comportamento deste alimento, predizer resultados e desenvolver pontos de controle do processo e do produto final. Foram utilizados grãos em casca com teor de umidade inicial de $12,25 \% \mathrm{X}_{\text {b.u. }}$ embebidos em água destilada nas temperaturas de $35,45,55,60$ e $65^{\circ} \mathrm{C}$ por um período de 15 horas. $\mathrm{O}$ aumento da temperatura resultou em acrescente taxa de absorção de água, caracterizando três regiões distintas, transiente, de transição e permanente. A densidade e o comprimento do grão se mantiveram constante ao longo da imersão nos diversos tratamentos térmicos, enquanto volume e raio foram influenciados pelas condições de gelatinização. O modelo proposto por Singh e Kulshrestha ajustou-se satisfatoriamente aos dados experimentais, $\mathrm{R}^{2}$ maiores que $97,2 \%$ e erro médio relativo entre os dados experimentais e preditos pelo modelo menores que $6 \%$.
\end{abstract}

\section{INTRODUÇÃO}

A produção mundial de arroz em 2013 chegou à marca de 590 milhões de toneladas. O Brasil foi o nono produtor mundial com 11 milhões de toneladas (FAO 2013).

A parboilização ocorre pela imersão do grão em casca em água aquecida, etapa de hidratação, para posterior indução do fenômeno da gelatinização, finalizando com a secagem e remoção da casca (Dors et al., 2009; Embrapa, 2013). De acordo com Elias et al. (2001) e a ABIAP (2013), as indústrias são orientadas a evitar a mistura de variedades, visto que, cada cultivar apresenta um comportamento peculiar diante das condições de imersão. Assim sendo, o conhecimento sobre as mudanças físicas e químicas provocadas pelo processo mediante a transferência de massa e calor são importantes para a garantia da qualidade do processo (Coutinho et al., 2010).

Múltiplos pesquisadores a cada ano exploram o processo de imersão de produtos agroindustriais (Maskan, 2001; Gowen et al., 2007; Cavariani et al., 2009; Botelho et al., 2010; Billiris et al., 2012), 
com a finalidade de avaliar o efeito do tempo e da temperatura sobre as inúmeras características do alimento (expansão, nutrição e absorção), tomando por alicerce uma abordagem matemática. O estudo matemático do processo de hidratação é comumente focado por modelos empíricos, com intuito de obter resultados relacionados à condição da cinética do processo (Shanthilal e Anandharamakrishnan, 2013). Os modelos empíricos apresentam aceitação devido sua facilidade de aplicação, manuseio dos dados e, por apresentar adequada análise do feito da hidratação em relação às variáveis do processo. A qualidade no ajuste dos dados preditos e experimentais também tem sido observada, por exemplo, Maskan (2002) com trigo $\left(\mathrm{R}^{2}=0,979\right)$, Botelho et al. (2010) para arroz $\left(\mathrm{R}^{2}=0,982\right)$, Quicazán et al. (2012) em soja $\left(\mathrm{R}^{2}=0,996\right)$ e Botelho et al. (2013) com milho $\left(\mathrm{R}^{2}=98,50\right)$.

A pesar dos diversificados estudos na área de hidratação, ainda são escassos os referentes ao processo de parboilização. Dentro deste contexto, este trabalho teve por objetivo uma modelagem matemática empírica do processo, e análise da variação das propriedades físicas do grão ao longo da imersão.

\section{MATERIAL E MÉTODOS}

Foi utilizado arroz em casca da variedade BR-IRGA 417, safra 2013. O teor de água inicial médio da amostra foi de $12,255 \% \mathrm{X}_{\mathrm{b} \text {.u. }}$, medido em triplicata.

\subsection{Procedimento experimental}

Cada ensaio utilizou proporção de 1:4 de arroz (em casca) em água destilada, sem agitação em banho termostático. As temperaturas exploradas foram de $35,45,55,60$ e $65 \pm 0,2^{\circ} \mathrm{C}$. Amostras de aproximadamente $20 \mathrm{~g}$ eram retiradas em intervalos de 30 minutos até totalizar 3 horas. Posteriormente, intervalos de 1 hora até a soma de 15 horas. Os ensaios ocorreram em triplicata. A umidade foi avaliada em base úmida descrita pela Equação 1.

$$
\% X_{b u}=\frac{X_{b u}-X_{b s}}{X_{b u}} \cdot 100
$$

Amostras de 30 unidades de grãos eram pesadas e destinadas à avaliação da densidade. O volume foi estimado pelo deslocamento de água em proveta. O raio e comprimento de cada amostra, constituída por 10 grãos, foram medidos empregando-se um paquímetro $( \pm 0,1 \mathrm{~mm})$.

\subsection{Modelagem Matemática do Processo de Hidratação}

Foi empregado o modelo empírico proposto por Singh e Kulshrestha (1987), Equação 3.

$$
U_{t}=U_{0}+\frac{C_{1} C_{2} t}{C_{2} t+1}
$$

A linearização da Equação 3 resulta na Equação 4, na qual $U_{t}$ é o teor de água; $U_{0}$ o teor de água inicial; $\mathrm{C}_{1}$ a capacidade de absorção de água; $\mathrm{C}_{2}$ a velocidade de absorção de umidade e t o tempo em 
horas.

$$
\frac{t}{U_{t}-U_{0}}=\left(\frac{1}{C_{1}}\right) t+\frac{1}{C_{1}-C_{2}}
$$

Ajuste do modelo: A qualidade do ajuste do modelo foi avaliada a partir do coeficiente de determinação $\left(\mathrm{R}^{2}\right)$, do erro médio relativo $(\mathrm{P})$ e estimativa do desvio padrão (SE). Os parâmetros $\mathrm{P}$ e SE foram calculados a partir das Equações 5 e 6, respectivamente. Nestas, E é o valor observado experimentalmente, $\hat{E}$ o valor predito pelo modelo, n o número de dados observados e GLR o grau de liberdade do modelo.

$$
P=\frac{100}{n} \sum \frac{|E-\hat{E}|}{E}
$$

$$
S E=\sqrt{\frac{\sum(E-\hat{E})^{2}}{G L R}}
$$

A qualidade da representação do modelo também foi verificada avaliando-se a raiz quadrada média do erro (RMSE) e a redução do chi-quadrado $\left(\phi^{2}\right)$, conforme as Equações 7 e 8 , respectivamente. Tal que, $\mathrm{N}$ identifica o número de observações e n o número de constantes.

$$
R M S E=\sqrt{\frac{\sum(E-\hat{E})^{2}}{N}}
$$

$$
\phi^{2}=\frac{\sum(E-\hat{E})^{2}}{N-n}
$$

\section{RESULTADOS E DISCUSSÃO}

\subsection{Análise da Variação de Umidade ao Longo do Processo de Hidratação}

A taxa do ganho de umidade do arroz parboilizado, para os ensaios até $60^{\circ} \mathrm{C}$, exibiram comportamento de absorção rápida no início do processo, divido em duas etapas, transiente e de transição, seguido por uma taxa mais constante, fase de regime permanente, conforme Figura 1. Kornarzyñski et al. (2002), Thakur e Gupta (2006), Perez et al. (2011) e Botelho et al. (2013) também verificaram este comportamento quando estudaram diferentes produtos agroindustriais. 


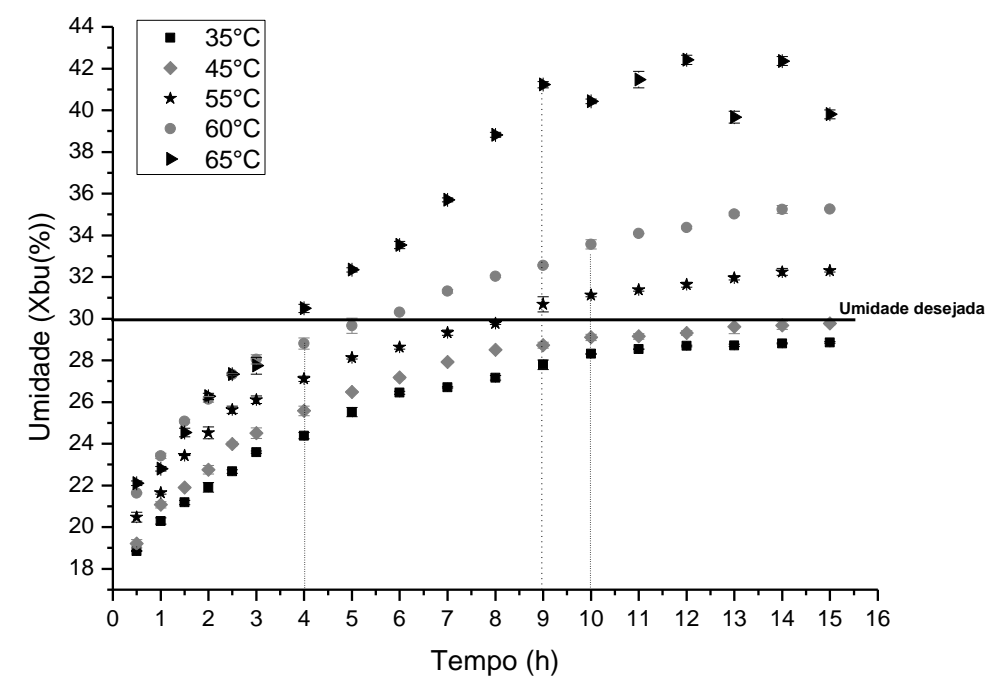

Figura 1 - Cinética de hidratação em função do tempo para as temperaturas de $35,45,55,60$ e $65^{\circ} \mathrm{C}$.

No intervalo de até 4 horas (região transiente), na faixa de 35 a $60^{\circ} \mathrm{C}$, houve um aumento médio de 116,02\% na umidade do produto em relação à inicial. De acordo com Gowen et al. (2007), Cavariani et al. (2009), Quicazán et al. (2012) e Ghafoor et al. (2014) o ganho de umidade instantâneo nas primeiras horas de processo é, possivelmente, devido à presença de fissuras e poros no alimento, tolerando maior ação capilar na superfície do pericarpo, induzindo a absorção de água. Segundo Maskan (2001), Turhan et al. (2002) a presença de espaços intermoleculares, admite que toda a estrutura do grão se hidrate. Quando o grão se aproxima da situação homogênea, a absorção tende a uma dinâmica pausada, intervalo de 4 a 10 horas (variação de 13,34\%). Com o prolongamento do processo, a taxa de absorção diminui acentuadamente até o equilíbrio, como verificado na faixa de 10 a 15 horas, havendo apenas 3,27\% de variação no ganho de água.

Junto à análise da tendência de absorção, o efeito da temperatura sobre o ganho de umidade foi estudado, que exibiu taxa de absorção quadrática $\left(\mathrm{R}^{2}\right.$ médio de $\left.83 \%\right)$ com o aumento da temperatura, o que foi comprovado pelas umidades de equilíbrio: 28,65;29,43; 31,78; 34,65 e 41,02 obtidas para $35,45,55,60$ e $65^{\circ} \mathrm{C}$, respectivamente. Pesquisadores como Addo et al. (2006), Fracasso et al. (2010) e Oliveira et al. (2013) também verificaram este comportamento e o atribuíram ao aumento do coeficiente de difusão que aumenta com temperatura, em função do aumento do nível de vibração das moléculas da água.

Segundo Kornarzyñski et al. (2002) e Han e Lim (2009) a temperatura ideal de hidratação para o processo de parboilização é a que proporciona os grãos atingirem a umidade necessária para ocorrência da gelatinização (almejada em 30\%, Figura 1) sem o rompimento da casca e, que minimizam a lixiviação de sólidos solúveis. Nas temperaturas de 35 a $60^{\circ} \mathrm{C}$ não houve rompimento da casca. Contudo, os ensaios de 35 e $45^{\circ} \mathrm{C}$ foram considerados inviáveis industrialmente, por necessitar de um período longo de hidratação, superior a15 horas o que possibilita a contaminação microbiana do produto. Os experimentos de 55 e $60^{\circ} \mathrm{C}$ ofereceram como tempo ótimo de processo 9 e 6 horas, 
respectivamente. Tais resultados são coerentes com os relatados por Bello et al. (2007) quando estudaram a relação de tempo e temperatura para a etapa de hidratação do processo de parboilização.

$\mathrm{O}$ ensaio a $65^{\circ} \mathrm{C}$, entretanto, Figura 1, apresentou o menor tempo relativo às condições idealizadas de umidade, ou seja, em 4 horas de imersão a massa total de arroz atingiu mais de $30 \%$ de seu peso em água. Com o prolongamento do processo nesta condição, nota-se que a absorção de umidade exibiu comportamento de regime transiente até 9 horas, resultando em um aumento de 236,40\% em relação ao teor inicial. Posteriormente, expôs conduta irregular, o que, de acordo com Resio et al. (2006) e Botelho et al. (2013) é atribuído ao fato dos grãos já terem alcançado as condições de gelatinização, quando acredita-se ter ocorrido a quebra de ligações de hidrogênio da região amorfa e fusão subsequente de matrizes cristalinas da estrutura do amido do arroz, proporcionando maior intumescimento do grão, consequentemente, facilitando o rompendo a casca. Com o rompimento da casca, tem-se o amolecimento/cozimento do produto, além de perda de sólidos.

\subsection{Análise das Variações Dimensionais do Grão ao Longo do Processo de Imersão}

Variação do volume: Steffe e Singh (1980) e Coutinho et al. (2007) enfatizaram que a variação do volume de grãos agropecuários ao longo do processo de hidratação é proporcional à quantidade de umidade absorvida. Na Figura 2, o volume do grão em relação ao ganho de umidade apresentou comportamento similar, exibindo uma região transiente (até 4 horas), de transição (4 a 10 horas) e permanente (ultimas 5 horas de processo) nos ensaios de 35 a $60^{\circ} \mathrm{C}$. Em testes a $65^{\circ} \mathrm{C}$, foi observado o mesmo comportamento, ou seja, variação do volume constante até 9 horas e, na sequencia comportamento desigual. Desta forma, o volume do grão de arroz está diretamente relacionado com sua capacidade de absorver umidade, porém a expansão é muito limitada devido à presença da casca.

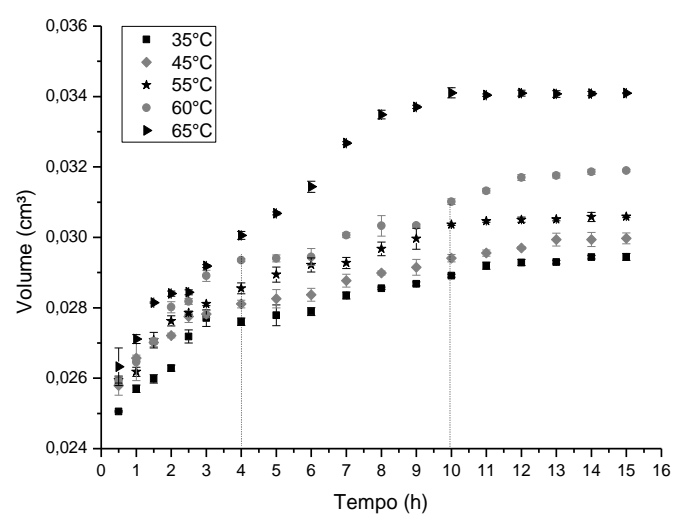

Figura 2 - Influência da combinação de tempo e temperatura de hidratação no volume do grão.

$\mathrm{O}$ aquecimento do sistema permitiu que o volume variasse de 0,024 a $0,033 \mathrm{~cm}^{3}$, somando $35,2 \%$ de alteração em relação à condição inicial. Ahromrit et al. (2006) não verificaram variação expressiva, apenas $1,6 \%$ quando hidrataram arroz Thai a $20^{\circ} \mathrm{C}$ por 2 horas. Perez et al. (2012), contudo, constataram que, em $1 \mathrm{~h} 30 \mathrm{~min}$ na faixa de 25 a $55^{\circ} \mathrm{C}$ o grão de arroz da variedade japônica variou de 0,017 a $0,023 \mathrm{~cm}^{3}$; ou seja, $35,3 \%$ de expansão. 
Variação da densidade: À medida que o tempo avançou durante o processo de hidratação a densidade do grão permaneceu praticamente constante, variação inferior a $1 \%$, correspondendo a um valor médio de $0,970496 \mathrm{~g} / \mathrm{cm}^{3}$. No trabalho anteriormente realizado por Perez et al. (2011), Shittu et al. (2009) e Corrêa et al. (2007) a densidade foi mantida constante para as variedades de arroz exploradas $\left(1,4 \mathrm{~g} / \mathrm{cm}^{3}\right.$ para a variedade japônica; $0,87 \mathrm{~g} / \mathrm{cm}^{3}$ para WAB-189 e 0,55g/cm ${ }^{3}$ para Jequitiba, respectivamente).

Variação do Raio e Comprimento: De acordo Muthukumarappan et al. (1992) Yadav e Jindal (2007) o monitoramento de parâmetros dimensionais de cereais/grãos é complexo, visto que, as dimensões oscilam com intensidade dentro da mesma variedade. É possível observar esta afirmação com base no desvio padrão de cada ponto amostral, ilustrado na Figura 3. No estudo de Yadav e Jindal (2007), estudando arroz da variedade kernel, os autores verificaram que alterações na dimensão radial são maiores em comparação com a longitudinal durante a imersão.

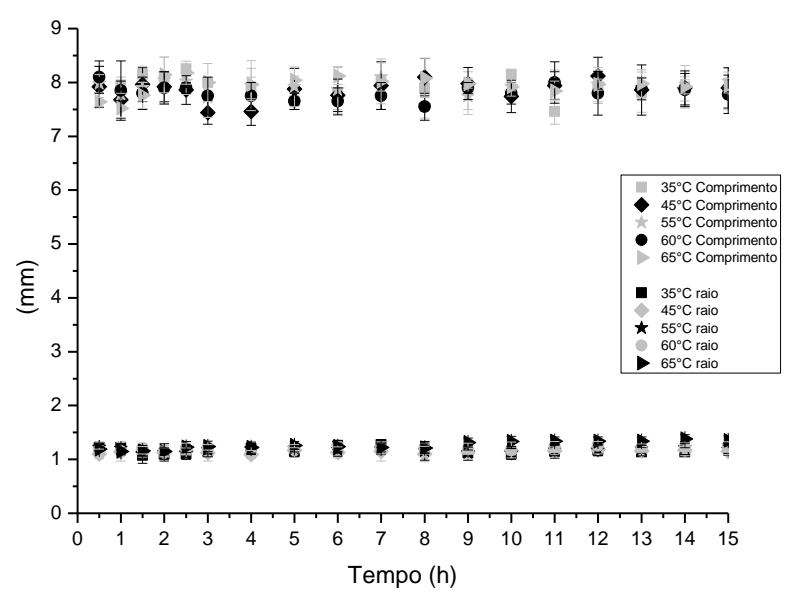

Figura 3 - Variação do raio e comprimento do arroz ao longo do processo de imersão.

Neste trabalho, o raio esboçou dinâmica simular em todos os ensaios até 8 horas de processo,

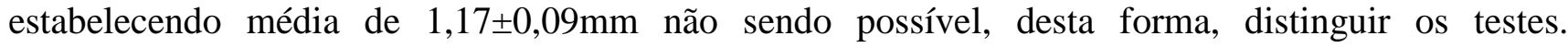
Posteriormente, na faixa de 9 a 15 horas, nota-se uma leve caracterização principalmente das tentativas em $65^{\circ} \mathrm{C}$, ou seja, o diâmetro dos grãos se sobressaiu aos demais ensaios e proporcionou médias equivalentes $(1,34 \pm 0,08 \mathrm{~mm})$. Este fato é justificado pelas variações morfológicas pelas quais o alimento é exposto nestas condições como, por exemplo, ocorrência da gelatinização, quebrando, assim, a resistência da casca em manter as formas originais do produto.

Ahromrit et al. (2006) verificaram que o comprimento de sua espécie de arroz sofreu maior influência do tratamento térmico que o próprio raio, contrariando os resultados obtidos nesta pesquisa, onde as alterações no comprimento permaneceram praticamente as mesmas durante o processo de hidratação, variando sempre na faixa de 7,2 a 8,5mm para todos os ensaios. No estudo de Thakur e Gupta (2006) e Shittu et al. (2012) a variação no comprimento de suas variedades de arroz foi de (7,011 a 7,285 e 7,76 a $8,38 \mathrm{~mm}$, respectivamente). 


\subsection{Modelagem Matemática do Processo de Imersão}

O modelo proposto por Singh e Kulshrestha (1987) foi aplicado com a finalidade de avaliar sua capacidade preditiva sobre as condições de hidratação exploradas para o arroz parboilizado. $\mathrm{Na}$ Figura 4, os dados experimentais são confrontados com os preditos pelo modelo matemático.

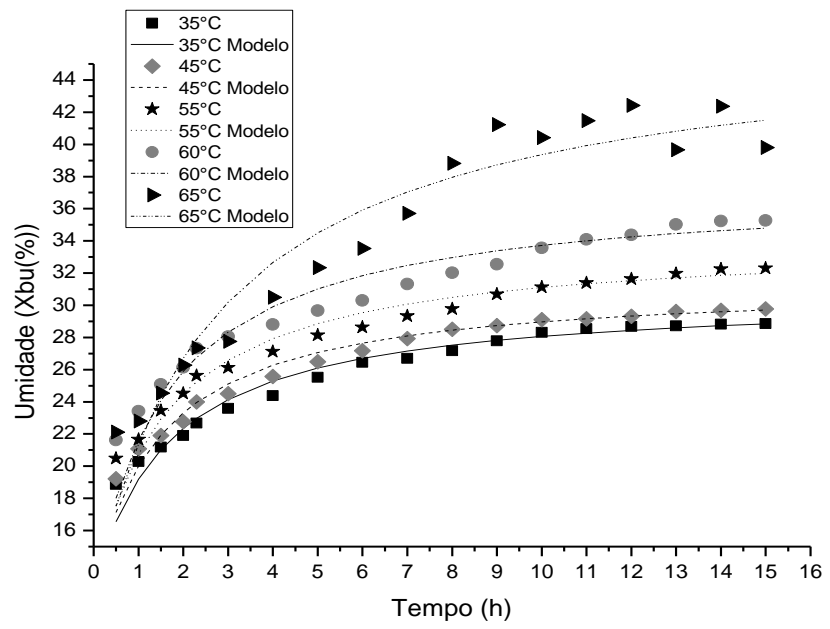

Figura 4 - Ajuste do modelo de Singh e Kulshrestha aos dados experimentais.

Os resultados estimados pelo modelo e os dados experimentais não coincidiram 100\%, deste modo, demais parâmetros estatísticos possibilitaram confirmar a qualidade do modelo quanto sua capacidade preditiva. Valores de $\mathrm{P}$ estiveram abaixo de $6 \%$, o que, segundo Resio et al. (2006) representam um ajuste aceitável do modelo. Valores de RMSE e SE, mencionados na Tabela 1, identificaram que os dados experimentais e os prenunciados variaram de forma significativa apenas nas temperaturas finais. Perez et al. (2011) quando estudaram imersão de arroz na faixa 25 a $55^{\circ} \mathrm{C}$ por meio do modelo de Peleg obtiveram valores de RMSE similares, alterando de 0.66 a 2.52 $\mathrm{X}_{\text {bu }}$. Botelho et al. (2010) na faixa de 55 a $75^{\circ} \mathrm{C}$ obtiveram valores de $\mathrm{SE}$ variando de 1,51 a $4,15 \mathrm{X}_{\mathrm{bu}}$. $\mathrm{O}$ teste de significância estatística Qui-quadrado $\Phi^{2}$ permitiu comparar a frequência dos dados observados com a frequência dos resultados estimados ao nível de 5\%. A análise de todos os ensaios permitiu manter a hipótese pautada, ou seja, os dados apresentaram concordância satisfatória $\left(\Phi^{2}<\Phi_{\alpha}^{2}=8,67\right)$.

Tabela 1 - Parâmetros e ajustes estatísticos do modelo em função da temperatura de hidratação.

\begin{tabular}{|c|c|c|c|c|c|c|c|}
\hline $\begin{array}{c}\text { Temperatura } \\
\left({ }^{\circ} \mathrm{C}\right)\end{array}$ & Parâmetros do Modelo & \multicolumn{5}{|c|}{ Ajustes do Modelo } \\
\hline 35 & $\mathrm{C}_{1}\left(\mathrm{X}_{\mathrm{bu}}\right)$ & $\mathrm{C}_{2}\left(\mathrm{~h} . \mathrm{X}_{\mathrm{bu}}\right)$ & $\mathrm{R}^{2}(\%)$ & $\mathrm{RMSE}\left(\mathrm{X}_{\mathrm{bu}}\right)$ & $\Phi^{2}\left(\mathrm{X}_{\mathrm{bu}}\right)$ & $\mathrm{SE}\left(\mathrm{X}_{\mathrm{bu}}\right)$ & $\mathrm{P}(\%)$ \\
\hline 45 & 18,416 & 0,696 & 99,66 & 0,6351 & 0,454 & 0,6736 & 1,674 \\
\hline 55 & 19,157 & 0,682 & 99,80 & 0,7045 & 0,558 & 0,7473 & 2,086 \\
\hline 60 & 21,787 & 0,641 & 99,61 & 0,8644 & 0,841 & 0,9168 & 2,317 \\
\hline 65 & 25,063 & 0,596 & 99,25 & 1,2264 & 1,692 & 1,3010 & 3,379 \\
\hline & 34,722 & 0,356 & 97,20 & 1,9247 & 4,168 & 2,0415 & 5,237 \\
\hline
\end{tabular}




\section{9 a 22 de outubro de 2014 \\ Florianópolis/SC}

Foi observado que os valores de SE, P, $\Phi^{2}$ e RMSE somaram com o aumento do aquecimento do sistema, evidenciando que o modelo ajustou melhor os dados experimentais para temperaturas inferiores, até $55^{\circ} \mathrm{C}$. Autores como Sopade et al. (2007), Górnicki et al. (2013) e Ghafoor et al. (2014) também observaram uma melhor adaptação dos modelos para temperaturas mais baixas, e atribuíram o evento à lixiviação de sólidos solúveis e gelatinização que ocorrem nesse tipo de processo com o aumento da temperatura, os quais não são contabilizados por modelos empíricos. Quanto ao modelo, o parâmetro $C_{1}$, Tabela 1 , é relativo à capacidade do arroz absorver umidade diante das condições testadas. A sensibilidade do parâmetro a temperatura sugere que, o efeito do aquecimento afeta o comportamento do grão quanto sua capacidade de absorção de forma positiva. Turhan et al. (2002), Cunningham et al. (2007) e Perez et al. (2011) verificaram a mesma relação; ou seja, com o aumento da temperatura aumenta a capacidade do alimento se hidratar. A constante $\mathrm{C}_{2}$, por sua vez, está relacionada com a velocidade de absorção de água. Logo, quanto menor $\mathrm{C}_{2}$, mais rápido será a taxa de absorção de umidade do produto.

\section{CONCLUSÕES}

O aumento da temperatura sistema permitiu a crescente taxa de absorção de água pelo grão. A densidade e o comprimento do arroz mantiveram-se constantes ao longo da hidratação, enquanto o volume e o raio foram influenciados pela gelatinização. O modelo proposto por Singh e Kulshrestha concordou satisfatoriamente com os dados experimentais para os ensaios a temperaturas até $55^{\circ} \mathrm{C}$, apresentando $\mathrm{R}^{2}$ maiores que $99 \%$ e erro médio relativo entre os dados experimentais e preditos menores que $3,5 \%$. Ensaios a $65^{\circ} \mathrm{C}$ tiveram os ajustes influenciados pelos eventos endotérmicos, $\mathrm{R}^{2}$ de $97,20 \%$.

\section{REFERÊNCIAS}

ABIAP- Associação de arroz Parboilizado do Brasil. Disponível em: <http://www.abiap.com. br/ArrozProcesso.php> Acesso em 17 de jul. de 2013.

ADDO, A.; BART-PLANGE, A.; DZISI, K. Water Absorption characteristics of Obatanpa and Mamaba Maize Hybrids (Zea mays). Int. J. Food Eng., v. 2, n. 3, p. 7, 2006.

AHROMRIT, A.; LEDWARD, D. A.; NIRANJAN, K. High pressure induced water uptake characteristics of Thai glutinous rice, J. Food Eng, v. 72, p. 225-233, 2006.

BELLO, M.; TOLABA, M. P.; SUAREZ, C. Factors affecting water uptake of rice grain during soaking. Lebensm. Wiss. Technol., v. 37, p. 811-816, 2004.

BELLO, M. O.; TOLABA, M. P.; SUAREZ, C. Water absorption and starch gelatinization in whole rice grain during soaking. $L W T$, v. 40, p. 313-318, 2007.

BILLIRIS, M. A.; SIEBENMORGEN, T. J.; WANG, Y.-J. Rice degree of milling effects on hydration, texture, sensory and energy characteristics. Part 2. Cooking using fixed, water-to-rice ratios. J. Food Eng, v. 113, p. 589-597, 2012.

BOTELHO, F. M., CORRÊA, P. C., GONELI, , M. A., BAPTESTINI, F. M. Análise da hidratação do arroz na parboilização. Ciênc. Tecnol. Aliment., v. 30, n. 3, p. 713-718, 2010. 